\title{
EDITORIAL
}

\section{Coronavirus Disease 2019 (COVID-19): An Opportunity to Understand the Health System}

\author{
M A FAIZ
}

As of 18 June 2020 Bangladesh recorded 102,292 cases and 1343 deaths since notification of the first case of COVID-19 on 8 March 2020. ${ }^{1}$ The Century's current pandemic, the Coronavirus Diseases 2019 (COVID-19) gave rise to a massive crisis in the health-socialeconomic arena across the globe, including in Bangladesh. Since the adoption of International Health Regulations (2005), global and country-specific preparations were initiated with a variable degree and importance by countries. ${ }^{2}$ Just a month before the origin of COVID-19 in December in China, Bangladesh prepared the National Action Plan for Health Security (NAPHS) 2020-24 through a series of year-long consultation. ${ }^{3}$ Immediately after the outbreak, Bangladesh prepared the six-point-plan 'to prevent and control of COVID-19 in Bangladesh to reduce impact on the health, wellbeing and economy of the country'. ${ }^{4}$ Soon we realized that successive adhoc actions could not prevent COVID-19 and unveiled system-wide deficiencies in public health and health care services, which was dominated by the private sector.

Bangladesh is a fertile field for infectious diseases. Other than good vaccination program we did not develop public health and clinical management capacity in infectious diseases despite a recurrent episode of infection having pandemic potential by Nipah infection. Our capacity of both 'stable platform' (examples, trained human resources, and infrastructure) and infusion of 'fast variables' (examples, quarantine, and isolation units) for fast emergency response in handling emerging infectious disease of pandemic potential was seriously lacking as well. ${ }^{5}$

Although a newly emerging infectious disease, the diagnostic capacity by RT-PCR, a labor-intensive procedure was started soon with rapid expansion to $>50$ centres within a short time. Judicious use of the test (could not minimize test on 'social reason') for infection control and suppression of transmission in an epidemic by quick 'isolation' of the highly contagious droplet mediated suspects and quarantine of the 'contacts' with 'confirmed' by WHO guidance was missing in the beginning and continued for sometime. ${ }^{6}$ Delay in getting the lab report along with inertia of mobilizing resources for the implementation of front line non-pharmaceutical public health interventions (social distancing, intense contact tracing, case isolation) exacerbated the situation quickly.

Bangladesh is a good example of having a rural community-based health care and track record of disaster management by engaging volunteers and community support groups. The absence of functional 'public' primary health care in urban areas became more visible during the pandemic. Bangladesh experienced recurrent outbreak by a dangerous pathogen, Nipah virus infection since 2000, having some experience which could not be translated as done by strong community engagement in the 'Kerala Model' to reduce the transmission of COVID-19 in Kerala, India. COVID-19 is an opportunity in disguise to strengthen the various components of health care services, including the 'community health system,' emergency system, and critical care with special note to dangerous infections in Bangladesh.

Early reports of dominant old-age predilection of vulnerability and respiratory involvement in COVID-19 soon became obsolete following evidence of thrombosis, cytokine surge, sepsis, acute kidney injury (AKI), multiorgan involvement, and inflammatory syndrome in children ('Kawasaki like syndrome'). ${ }^{7}$ Till specific antivirals are recommended, we have to rely on providing efficient, supportive care to patients taking precautions by health care professionals. In a state-of-art facility of New York, 257/1150, 22\% were critically ill, 79\% required invasive mechanical ventilation of whom 28-day follow up could detect $39 \%$ death, and $37 \%$ still on ventilation at the time of reporting. ${ }^{8}$ We have very limited capacity confined to a few big cities for providing intensive care in such a dangerous pathogenic virus infection COVID- 
19. In Bangladesh, so far, few patients who received mechanical ventilatory support could survive, meanwhile successful use of a high-flow nasal cannula (HFNC) has been found to be useful in improving oxygenation.

Most affected populations in COVID-19 were mild but considering the speed and number of the population to be affected with variable intensity with the proportion that would require stepwise support by oxygen delivery, and respiratory support could not be reliably forecasted hindering quick capacity in providing care in the pandemic surge. Early in the pandemic instruction for separate 'flu corner' in different tiers of the hospital was made. Enthusiastic media coverage about less dangerous 'flu' like illness following COVID-19 soon proved to be wrong.

Infectious diseases were not a priority in health agenda, forgetting the importance of basic principles of infection prevention and control (IPC), infectious diseases in health human and infrastructural resources. Separate dedicated COVID-19 hospitals were set up for clinical service provision, which was contradictory to the 'team approach' of care. Stepwise expansion of isolation units and hospital for the care of COVID-19 in the capital, division, and district headquarters were made, multisectoral committees were formed and attempt to involve non-government hospitals were made. The utilization of telemedicine was quickly used. Inspite of all attempts, both COVID-19 and non-COVID patients were found to be not getting adequate care exacerbated by public perception of transmission mechanism causing irrational and at times, inhuman behavior towards suspects, close relatives, neighbors and deceased. A country wide network of medical colleges and district hospitals gave an opportunity to involve the faculty and staff in the care of COVID-19 and nonCOVID patients in the same hospital with proper IPC including appropriate personal protective equipment (PPE), triage and separation into 'COVID,' 'suspect' and non-COVID.

Mobilization of resources for emergency care starting from community, transfer to a health facility, dedicated emergency room considering highly infectious pathogen, triage, segregated stepwise care keeping in mind necessity of oxygen delivery, evolving pathology directed investigations guiding management including advanced care were mobilized in countries with enough preparation and resources.

The World Health Organization quickly prepared a road map of COVID research to develop drug, vaccine in fast track, and conducting a large number of relevant research having recent experience from Ebola infection. ${ }^{9}$ Till effective usable vaccines are available, we have to rely on public health measures and effective adherence to them to adjust with 'new normal.'

(J Bangladesh Coll Phys Surg 2020; 38: 1-2) DOI: https://doi.org/10.3329/jbcps.v38i0.47437

\section{Prof. MA Faiz}

Professor of Medicine (Retired), Former Director General of Health Services.

Scientist, Dev Care Foundation, Dhaka, Bangladesh, E-mail:drmafaiz@gmail.com

\section{References:}

1. Institute of Epidemiology, Disease Control and Research. https://www.iedcr.gov.bd/.

2. WHO. International Health Regulations. Second Edition. 2005.

3. MOHFW. National Action Plan for Health Security (NAPHS) Bangladesh 2020-2024. 2019.

4. MOHFW. National Preparedness and Response Plan for COVID-19, Bangladesh. 2020.

5. Kruk ME, Myers M, Varpilah ST, Dahn BT. What is a resilient health system? Lessons from Ebola. Lancet. 2015;385(9980):1910-2.

6. WHO. COVID-19 strategy update. April, 14, 2020. https:/ /www.who.int/docs/default-source/coronaviruse/covidstrategy-update-14april2020.pdf.

7. WHO. Clinical management of COVID-19. Interim guidance. 2020.

8. Cummings MJ, Baldwin MR, Abrams D, Jacobson SD, Meyer BJ, Balough EM, et al. Epidemiology, clinical course, and outcomes of critically ill adults with COVID-19 in New York City: a prospective cohort study. Lancet. 2020;395(10239):1763-70.

9. WHO. A coordinated global research roadmap: 2019 novel coronavirus. 2020. 